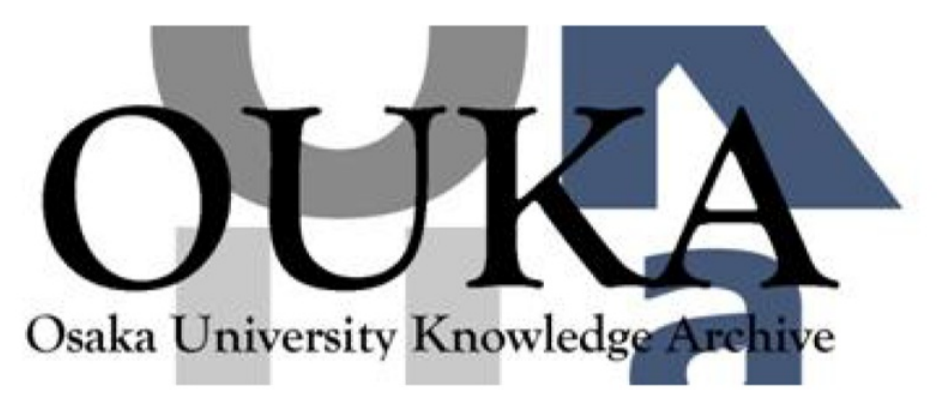

\begin{tabular}{|c|l|}
\hline Title & $\begin{array}{l}\text { Projection-based visualization of tangential } \\
\text { deformation of nonrigid surface by deformation } \\
\text { estimation using infrared texture }\end{array}$ \\
\hline Author(s) & $\begin{array}{l}\text { Punpongsanon, Parinya; Iwai, Daisuke; Sato, } \\
\text { Kosuke }\end{array}$ \\
\hline Citation & Virtual Reality. 19(1) p. 45-p. 56 \\
\hline Issue Date & $2015-03$ \\
\hline oaire:version & AM \\
\hline URL & https://hdl.handle. net/11094/81841 \\
\hline rights & \\
\hline Note & \\
\hline
\end{tabular}

Osaka University Knowledge Archive : OUKA

https://ir. Library. osaka-u. ac. jp/

Osaka University 


\title{
Projection-based Visualization of Tangential Deformation of Nonrigid Surface by Deformation Estimation Using Infrared Texture
}

\author{
Parinya Punpongsanon - Daisuke Iwai - Kosuke Sato
}

Received: date / Accepted: date

\begin{abstract}
In this paper, we propose a projection-based mixed reality system that visualizes the tangential deformation of a nonrigid surface by superimposing graphics directly onto the surface by projected imagery. The superimposed graphics is deformed according to the surface deformation. To achieve this goal, we develop a computer vision technique that estimates the tangential deformation by measuring the frame-by-frame movement of an infrared (IR) texture on the surface. IR ink, which can be captured by an IR camera under IR light, but is invisible to the human eye, is used to provide the surface texture. Consequently, the texture does not degrade the image quality of the augmented graphics. The proposed technique measures individually the surface motion between two successive frames. Therefore, it does not suffer from occlusions caused by interactions and allows touching, pushing, pulling, and pinching, etc. The moving least squares (MLS) technique interpolates the measured result to estimate denser surface deformation. The proposed method relies only on the apparent motion measurement; thus, it is not limited to a specific deformation characteristic, but is flexible for multiple deformable materials, such as viscoelastic and elastic materials. Experiments confirm that, with the proposed method, we can visualize the surface deformation of various materials by projected illumination, even when the user's hand occludes the surface from the camera.
\end{abstract}

Keywords Projection-based mixed reality · User interaction · Deformable surface

P. Punpongsanon · D. Iwai · K. Sato

Osaka University, 1-3 Machikaneyama, Toyonaka, 560-8531

Osaka, Japan.

Tel: +81-6-6850-6372

Fax: +81-6-6850-6341

E-mail: parinya@sens.sys.es.osaka-u.ac.jp

\section{Introduction}

Projection-based mixed reality (MR) refers to a technique in which computer graphics are projected on an actual object to augment its appearance without refabricating the object or requiring any user-worn equipment (Raskar et al, 2001, 2006). Compared with MR techniques that use video/optical see-through displays, projection-based MR is more suitable for multi-user scenarios because it provides users with a natural field of view without requiring them to wear or hold any additional physically constraining devices (Bimber and Raskar, 2005). This enhances communication, particularly the sharing of augmented information during collaborative tasks (Bluteau et al, 2005; Ni et al, 2011). Projection-based MR can be integrated into many application fields such as simulation (Jones et al, 2013), designer support (Bandyopadhyay et al, 2001; Rivers et al, 2012), and entertainment (Mine et al, 2012; Saakes et al, 2010). However, current technologies typically support only rigid objects and are not applicable to deformable or malleable objects. Note that in this study, we refer to "deformation" as a tangential deformation of a surface rather than a bump deformation.

The projection-based augmentation of deformable objects has a broad range of application possibilities. For example, visualizing surface deformation can improve the study of fluid dynamics by allowing students to touch a deformable object and observe its deformation. It can also support a graphical designer in creating deformed graphics with a free-form tangible interface on which the deformation is visualized by projection. Moreover, projection-based MR with deformation has the potential to enhance advertisements for nonrigid products in a retail store by allowing customers 


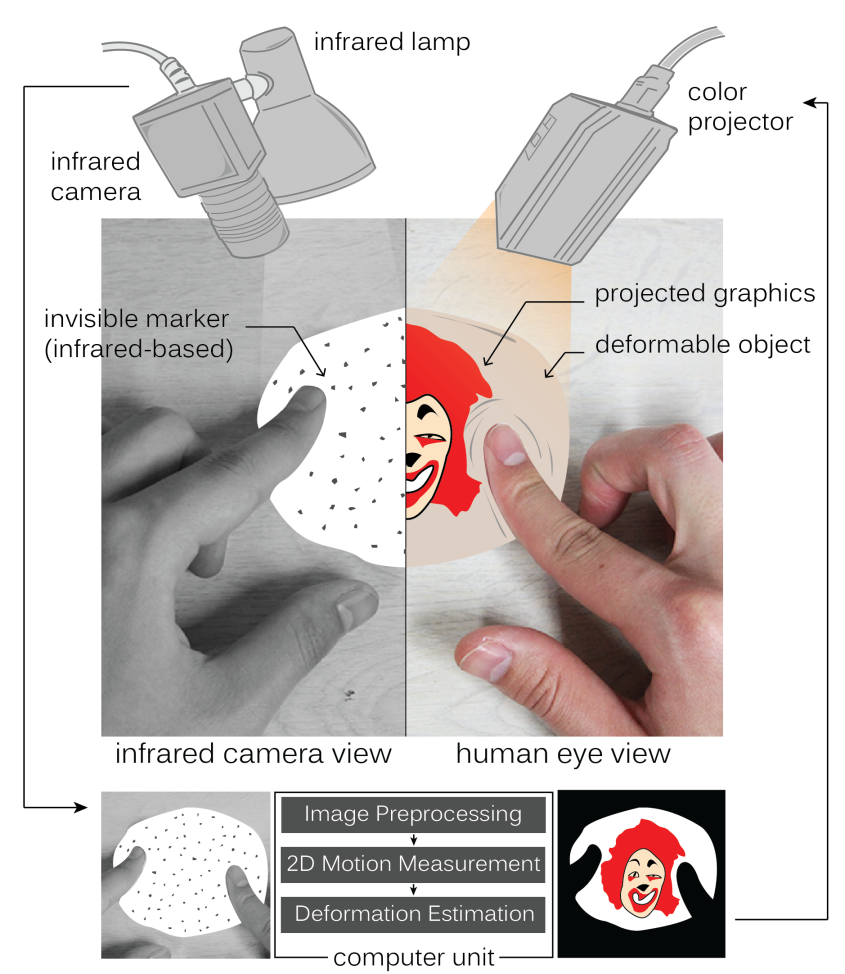

Fig. 1 System concept.

to interact with the projected ads on the product by deforming (e.g., pressing and bending) the product.

The aim of this study is to provide a projectionbased MR system that visualizes tangential deformation on a surface by deforming the projection graphics to match the actual surface deformation in real-time. To achieve this goal, we propose a computer vision technique to measure the tangential motion of visual feature points on a deformable surface. Because of occlusions and non-uniformity of the surface texture density, we cannot measure the motion in some areas of the surface. Therefore, we interpolate the measured result using the moving least squares (MLS) method (Schaefer et al, 2006) to estimate the final deformation information. Because our feature tracking method relies only on the measurement of apparent surface motion, it is not limited to a specific deformation characteristic. The proposed method is flexible for multiple deformable materials, such as viscoelastic and elastic materials. To avoid tracking failures caused by occlusions, we propose tracking visual features individually on the surface between two successive frames, rather than through an entire capture sequence. We refer to this technique as within-two-frames feature tracking. We use infrared (IR) ink, rather than visible ink, to provide a surface texture to make the surface motion detectable by an IR camera without degrading the image quality of visible projected graphics. Figure 1 shows an overview of the proposed concept.

The primary contributions of this work can be summarized as follows:

- Image-based approach for tangential deformation estimation of various materials;

- Within-two-frames feature tracking method for measuring tangential motion of deformation surface, which is robust against occlusions caused by user interactions;

- IR texture on a surface for stable feature detection under visible projector illumination;

- Combination of sparse feature tracking and MLSbased interpolation for real-time deformation estimation.

\section{Related Work}

Projection-based MR, which provides virtual graphics to augment physical objects, can be applied to enrich interactive content (Bandyopadhyay et al, 2001; Raskar et al, 2006; Mine et al, 2012), enhance appearances (Bimber and Iwai, 2008; Iwai and Sato, 2010; Shimazu et al, 2011) and create a deformed illusion on rigid objects (Hisada et al, 2006). To date, projection-based MR researchers have primarily focused on rigid objects, and little work has been conducted on nonrigid objects.

Pioneering work on projection-based MR for a nonrigid surface has been performed by Piper et al. (Piper et al, 2002). Their system consisted of a depth sensor, a projector and clay. Users interact with the clay (e.g., building a terrain map) onto which the projector superimposes depth-related imagery, such as pseudo-color representation of the height information of the map. Recently, Steimle et al. proposed estimating the bump deformation of a sheet of paper by using a depth sensor with depth-related imagery projected onto it (Steimle et al, 2013). Other work has applied projection-based MR to a medical surgery scenario, in which the navigation information is projected directly onto a patient's body (Kocev et al, 2013). These studies have focused on the bump deformation of a projection surface rather than tangential deformation.

We apply a visual feature tracking approach to measure tangential deformation. Several studies have tackled this issue. Pilet et al. presented a method that detects and tracks deformable objects in monocular image sequences (Pilet et al, 2008), in which wide baseline matching is applied to find feature correspondences between a reference image and a captured deformed object. However, this method has a high computational cost. Other studies have proposed real-time deforma- 
tion estimation methods using richer information, i.e., both depth images and visual features (Haouchine et al, 2012; Shimizu et al, 2013). These methods work for global deformation, by which the appearance of a small area around a feature point, and consequently the feature vector, does not change significantly. Thus, the global deformation does not introduce distraction to the process of finding feature correspondences. On the other hand, these methods do not work for local deformation, which changes the appearance of a small area dramatically, making feature matching impossible.

Kamiyama et al. proposed GelForce, method for estimating local deformation (Kamiyama et al, 2005). This method estimates the magnitude and direction of the tangential force of a deformable silicone surface by measuring the deformation of red and blue markers embedded in the object. However, the materials that can be used in the system are limited because the method requires a physical model of the material as well as a dense marker array on the entire surface. Ito et al. proposed method for estimating the force of a dexterous handling robot (Ito et al, 2014). This method estimates the orientation as well as direction of tangential force by measuring the displacement of the center dot using the contact area and contact depth of a fluid-type elastic touchpad. Although the method achieved the estimation of the rotation and direction of the tangential force, it is designed for a specific application, i.e., contact force detection in a robot, and cannot be used for our applications. We aim to estimate local tangential deformation of various deformable surfaces (e.g., elastic, viscous, and viscoelastic materials) without assuming any specific physical models while the marker density is not related to the size of the surface.

An alternative method for detecting the local deformation is to apply indirect sensing techniques. Sato et al. detected the natural changes in the photoelastic property in order to detect the place where transparent silicone was deformed (Sato et al, 2009). Heo and Lee used the normal force of a finger to estimate the shear force applied to the touch screen surface (Heo and Lee, 2013). Other works have detected bump deformation of elastomer using a depth from structure light (Follmer et al, 2011; Matoba et al, 2012) or using an illumination of different position red, green, blue light (Johnson et al, 2011). These methods have focused on the bump deformation from touch (or pressure) to reconstruct the input shape, and cannot applied to the tangential deformation of a dilatation surface.

We propose the within-two-frames feature tracking method for the estimation of local tangential deformation. We assume that surface deformation between two successive frames can be regarded as global de- formation; thus, feature correspondences between these frames can be determined. The proposed method measures feature displacement between the previous and the current frame, and adds it to the estimated deformation result from the previous frame. A previous study (Wu et al, 2012) has shown that the summation of frame-by-frame displacement of a point over a long sequence (i.e., an Eulerian-based approach) is equivalent to the displacement of the point from the first to the last frame of the sequence (i.e., a Lagrangian-based approach).

Visual features can be moved in any arbitrary directions on a deformable surface exhibiting both global and local deformations. In other words, the features are not always evenly distributed on surfaces, and there may be a region without any features, for which the feature tracking method fails. Therefore, we need to interpolate the measured displacement results by the within-two-frames feature tracking. For this purpose, we apply an MLS method, which is a sophisticated computer graphics technique designed for generating realistic deformation of a graphical character (Schaefer et al, 2006). MLS produces smooth deformations to the entire graphic character using a small number of control points.

As discussed in Section 1, there are many application fields that require deformation visualization for various materials. To realize such applications, we need a deformation estimation method of a nonrigid surface that can be applied for different materials in real-time. Several deformation visualization methods have been proposed, such as tracking visual features on the surface from overhead image sensors (Uchiyama and Marchand, 2011; Uchiyama and Saito, 2011; Fujimoto et al, 2014), or under the surface (Sato et al, 2009; Vlack et al, 2005), and embedded sensors (Heo and Lee, 2013). However, these methods only work for specific materials and/or cannot work in real-time. Our method enables users to visualize the tangential deformation of different deformable materials and work with them in real-time, independent of the surface size, simply by painting or embedding IR ink on the deformable materials.

\section{Deformation Estimation Technique}

In this section, we present our tangential deformation estimation method. Figure 2 shows the process flow of the proposed technique. The deformable surface with IR markers is captured by an IR camera. The surface detection module finds the target region in the captured image. The motion measurement module calculates feature points and their correspondences between 


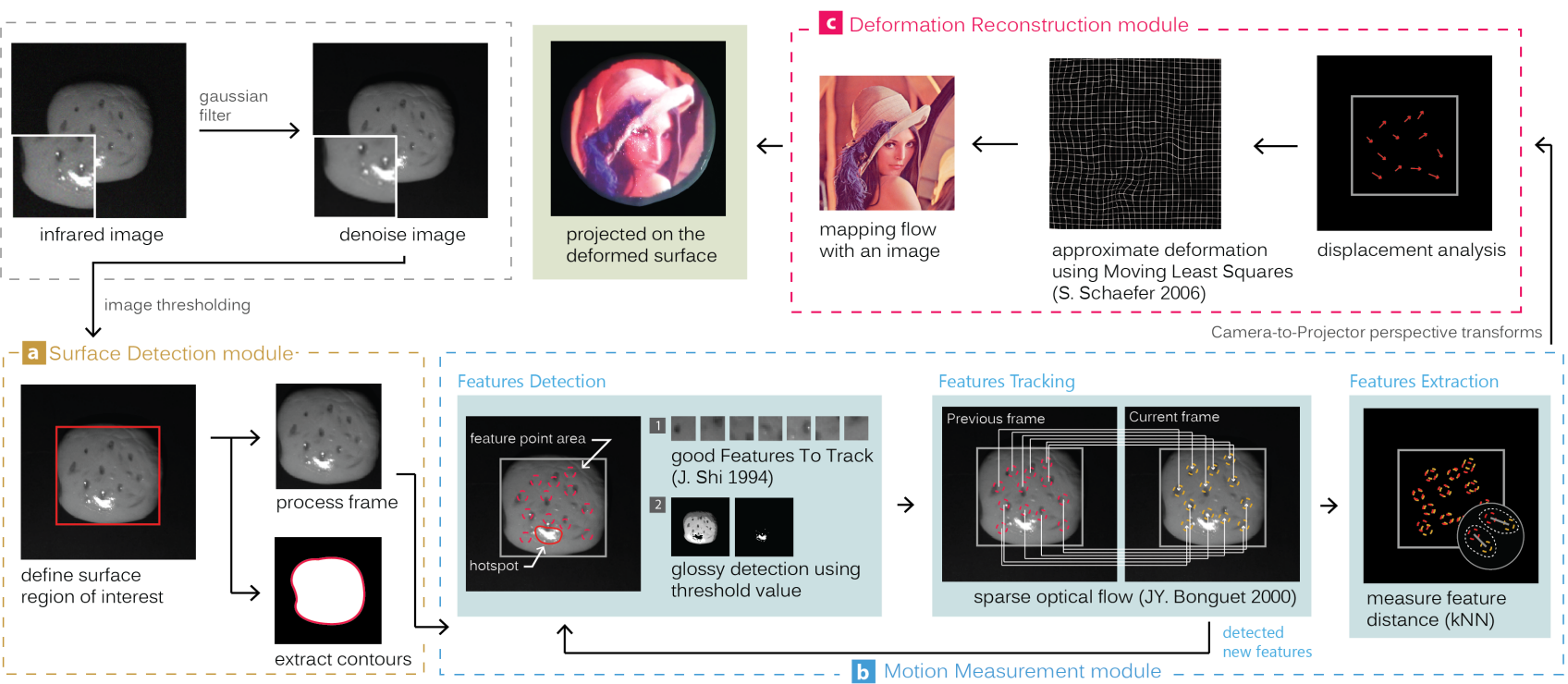

Fig. 2 Process flow framework: (a) surface detection, (b) motion measurement, and (c) deformation reconstruction.

two successive frames. Thereafter, the deformation reconstruction module estimates the deformation of the surface by interpolating the measured displacement information using MLS, and adds the estimation result to that from the previous frame.

\subsection{Surface Detection}

In this research, we assume that the background of our system, as well as the IR ink (Section 4), absorb IR light. Therefore, only the surface of the deformable object exhibits high intensity under uniform IR illumination in a captured IR image. We detect the surface region of the deformable object by finding the largest bright region in each captured image. We define a square region containing the surface boundary as a region of interest (ROI). Rather than using the entire captured image, the ROI is used as a working area in the following process to make it faster and ensure robustness against image noise occurring outside the ROI.

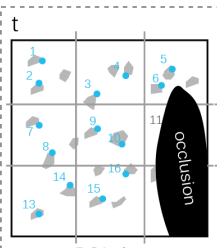

ROI view

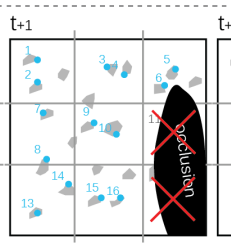

(a)

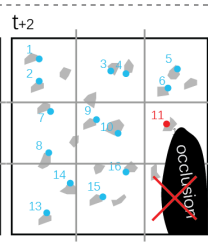

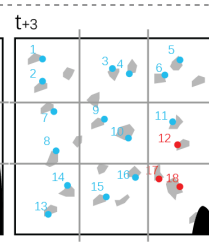

(2)

\subsection{Motion Measurement}

We propose the within-two-frames feature tracking method to measure spatially varying movements of feature points on a deformable surface. The proposed method is designed to avoid tracking failures caused by occlusions, which are unavoidable when features are tracked through an entire captured sequence. Our method computes feature correspondences between two successive frames. If some feature points are occluded, we ignore them, and measure only non-occluded points. Therefore, the tracking errors caused by occlusions are not accumulated.

For stable motion measurement, feature points should be distributed evenly over the surface. Therefore, we divide the ROI into $m \times m$ blocks and measure the fixed number of feature points, $\alpha$, in each block. We define the total number of feature points $K$, which is then divided by the number of blocks to obtain $\alpha$. We apply a simple feature detector, i.e., Good Features To Track (Shi and Tomasi, 1994), in our system.

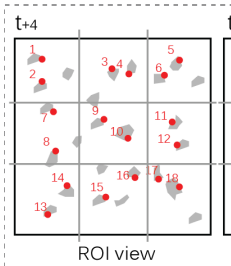

$\mathrm{ROI}$ view

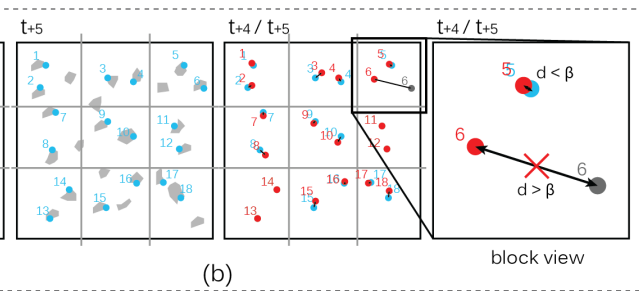

(b)
- detected feature corresponding point $\bullet$ ignore point

Fig. 3 Within-two-frames technique used to measure surface motion. The feature point correspondences are calculated in each block individually. (a) If a block is occluded, the block is ignored until the surface in the block reappears. (b) The proposed method avoids matching errors by finding unusual correspondences from the displacement of each feature correspondence. 


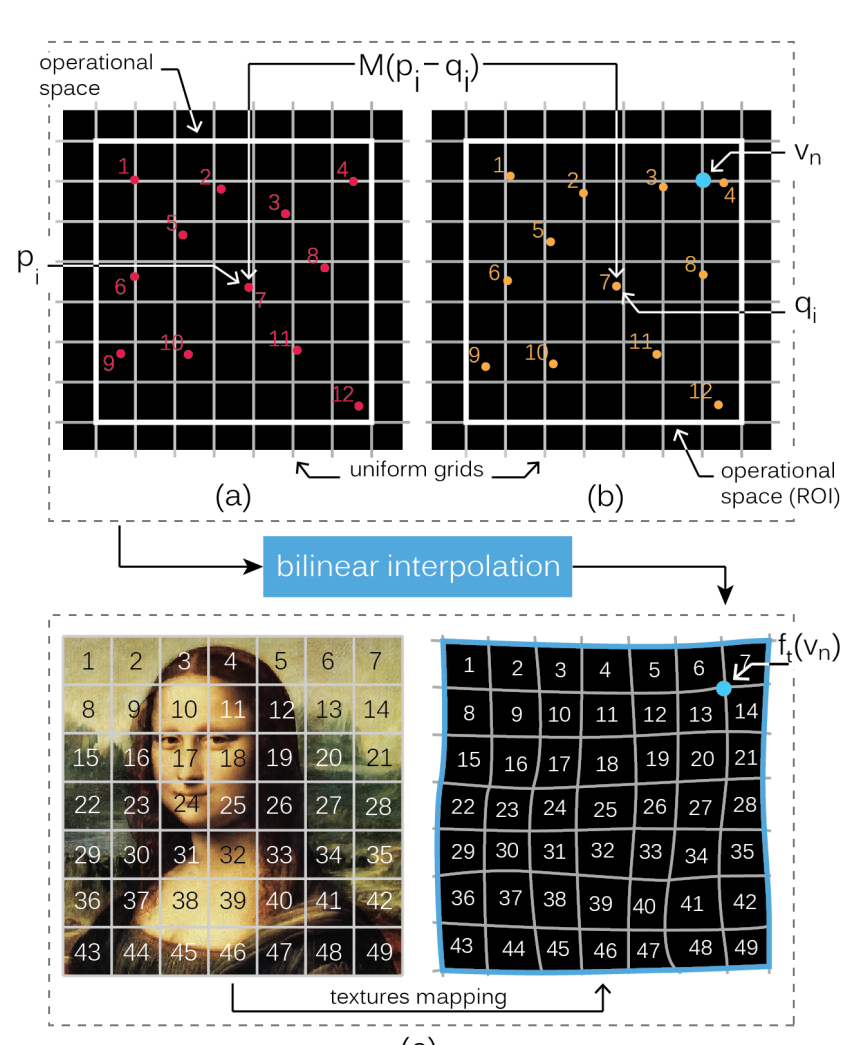

(c)

Fig. 4 (a) Transformation matrix $M$ maps between detected feature points $p_{i}$, (b) feature correspondences $q_{i}$, and (c) texture image mapped to the deformation estimation result at each vertex $f_{t}\left(v_{n}\right)$.

In the feature tracking process, we find a feature point in the current frame, i.e., the corresponding point that corresponds to a point in the previous frame, i.e., the original point. The corresponding point is determined as the feature point that is the nearest to the original point. The proposed method determines the feature points and their correspondences individually for each block. If a block is completely occluded, it is not possible to determine the feature points and their correspondences. In this case, we ignore that particular block and attempt to measure the surface motion when the surface reappears in the block (Fig. 3(a)). Our method also avoids matching errors by finding unusual correspondences. We consider a feature correspondence as an unusual correspondence when the displacement value $d$ is greater than a threshold value $\beta$. A threshold value $\beta$ is specified in advance based on the assumption that, within 25 frames, the feature point should not move more than 0.3 centimeters or 10 pixels. We exclude such features and their correspondences from the motion measurement (Fig. 3(b)).

Specular reflection on a deformable surface is problematic: when some regions of a surface exhibit specular reflection of the illuminated IR light, they exhibit bright spots close to white in a captured image. This causes a matching error and results in a tracking failure. To solve this problem, we exclude the pixels that have brightness values greater than a threshold value $\gamma$ from the motion measurement. A threshold value $\gamma$ is calculated by (maximum brightness value - minimum brightness value) $\times 0.8$ based on a method proposed by Chang and Tseng (Chang and Tseng, 2010).

\subsection{Deformation reconstruction}

In general, dense feature tracking realizes an accurate measurement of the surface motion; however, it requires a rich texture covering the entire surface. Such rich textures do not always appear on a deformed surface because the texture moves unevenly according to the deformation and may be occluded by user interaction. Therefore, we apply an interpolation technique (i.e., MLS) to the motion measurement result to estimate the final deformation.

MLS was originally proposed to generate in realtime realistic deformations of a graphic character using only a small number of control points. Smooth deformations are produced from the displacement of each control point in each frame. We apply this method to the feature tracking result rather than using the movement of predefined control points.

Unlike previous MLS approaches, we are concerned with the ROI rather than the entire image. Because the size of the ROI changes according to the surface deformation, we geometrically transform the feature tracking results in such a way that the ROI of each frame corresponds to a reference, i.e., the ROI of the first frame. The transformed ROI is referred to as the operational space in the following. Estimation of the deformation at every location in an operational space requires significant computational resources. Therefore, we calculate the deformation at sparse but uniformly distributed points in the space. We assign a uniform grid to the operational space, and the MLS computation is performed at the grid vertices. The number of uniform grids is related to the deformation quality and computational resources. We assign a number of uniform grids based on the size of the deformable object. Our preliminary experiment shows that a $6 \times 6$ uniform grid per square centimeter $\left(\mathrm{cm}^{2}\right)$ provides optimal quality and computational resources.

First, we geometrically transform a set of feature points and their correspondences from each ROI to the operational space. In Fig. 4 , let $p_{t}^{i}$ and $q_{t}^{i}$ be the original points and their correspondences, respectively, at time $t$, where $i$ indicates the index of each point. Then, we define the deformation at each grid vertex $v_{n}(n=$ 


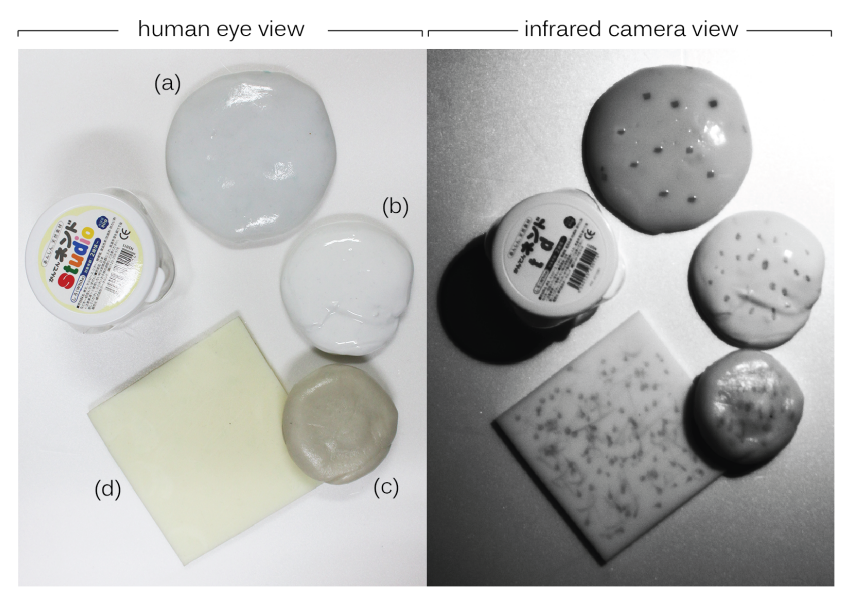

Fig. 5 Human eye view (left) and IR camera view (right) of deformable objects painted or embedded with invisible inkpainted fabrics: (a) commercial silly putty, (b) slime (sodium borate + PVA) embedded with invisible particles, (c) elastic clay, and (d) polyurethane-based gel sheet painted with invisible ink.

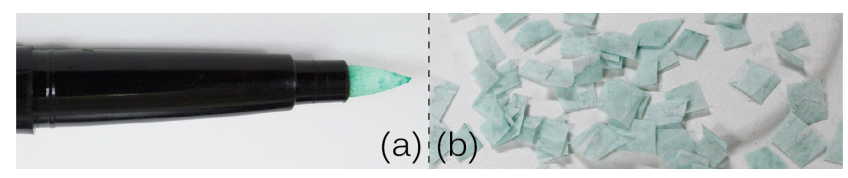

Fig. 6 (a) Invisible ink with high absorption in the IR region, and (b) spectral selective particles, i.e., IR invisible ink painted on non-woven fabrics.

$1, \ldots, N)$ by solving a transformation $M$ that maps $p_{t}^{i}$ to $q_{t}^{i}$ between each of the two successive frames (see the solving formulation of $M$ in (Schaefer et al, 2006)):

$f_{t}\left(v_{n}\right)=\min _{M} \sum_{i} w_{t}^{i}\left(v_{n}\right)\left|M\left(p_{t}^{i}-q_{t}^{i}\right)\right|^{2}$,

where position $f_{t}\left(v_{n}\right)$ is the estimation result of $v_{n}$ at time $t$ and $w_{t}^{i}\left(v_{n}\right)$ is the weight function of the distance between $v_{n}$ and $p_{t}^{i}$ defined as

$w_{t}^{i}\left(v_{n}\right)=\frac{1}{\left|p_{t}^{i}-v_{n}\right|^{2}}$.

The sum of the estimation result from each of the two successive frames will yield the total deformation estimation result. Consequently, the total deformation result at $v_{n}$ from $t=1$ to $T$ is computed as

$F_{T}\left(v_{n}\right)=f_{T}\left(v_{n}\right)+\sum_{t=1, \ldots, T-1} f_{t}\left(v_{n}\right)$.

To visualize the deformation estimation result, we geometrically transform the operational space to the projector coordinate. Subsequently, we map projection graphics to the deformation estimation result. The deformed graphics are projected on the surface using a projector.

\section{Deformable Objects}

Our system can use materials that have various deformation properties, such as viscoelastic or elastic materials. Several examples of deformable objects are shown in Fig. 5(a), including slime ${ }^{1}$, elastic clay (NENDO Studio clay), a polyurethane-based gel sheet (EXSEAL Hitohada), and silly putty (Crazy Aaron Enterprises). Viscoelastic materials exhibit a time-dependent strain when the deformation is performed. Elastic materials change shape under force and recover their shapes when the force is released. These materials allow for several interactions, such as touching, pinching, squeezing, kneading, and pulling or stretching.

Deformable materials for our application must have uniformly white, or at least non-textured surfaces for projector illumination. This is difficult considering that our deformation estimation method requires a surface texture which can be used for vision-based feature detection. To make a non-textured deformable surface applicable for our deformation estimation method, we use spectral selective ink, called IR ink (Fig. 6(a)). The IR ink is invisible to the human eye, having high absorption only in a near-IR wavelength (between 793 $\mathrm{nm}$ and $820 \mathrm{~nm}$ ). We use two approaches for applying IR ink to make a deformable material that has a non-textured surface applicable for our deformation estimation method.

First, we paint the IR ink directly on the deformable surface. The IR ink is oil-based; thus, it is durable after application to the surface. In particular, this approach is appropriate for matte materials, such as polyurethanebased gel sheets and elastic clay.

Second, we embed pieces of fabric soaked in IR ink into a deformable object approximately $0.1-0.2 \mathrm{~cm}$ under the surface (Fig. 6(b)). This approach is particularly effective for objects having glossy surfaces, such as slime and silly putty, which are inappropriate for the painting approach. Owing to the IR wavelength being wider than visible wavelength, the embedded particles are still visible in an image captured by an IR camera. The fabric used in our system is non-woven, and is matte and durable when soaked in ink. The fabric is particularly suitable because it does not disperse IR ink into the deformable objects.

Figure 5(b) shows the IR image of Fig. 5(a).

\footnotetext{
1 see http://en.wikipedia.org/wiki/Flubber (material) for more information.
} 

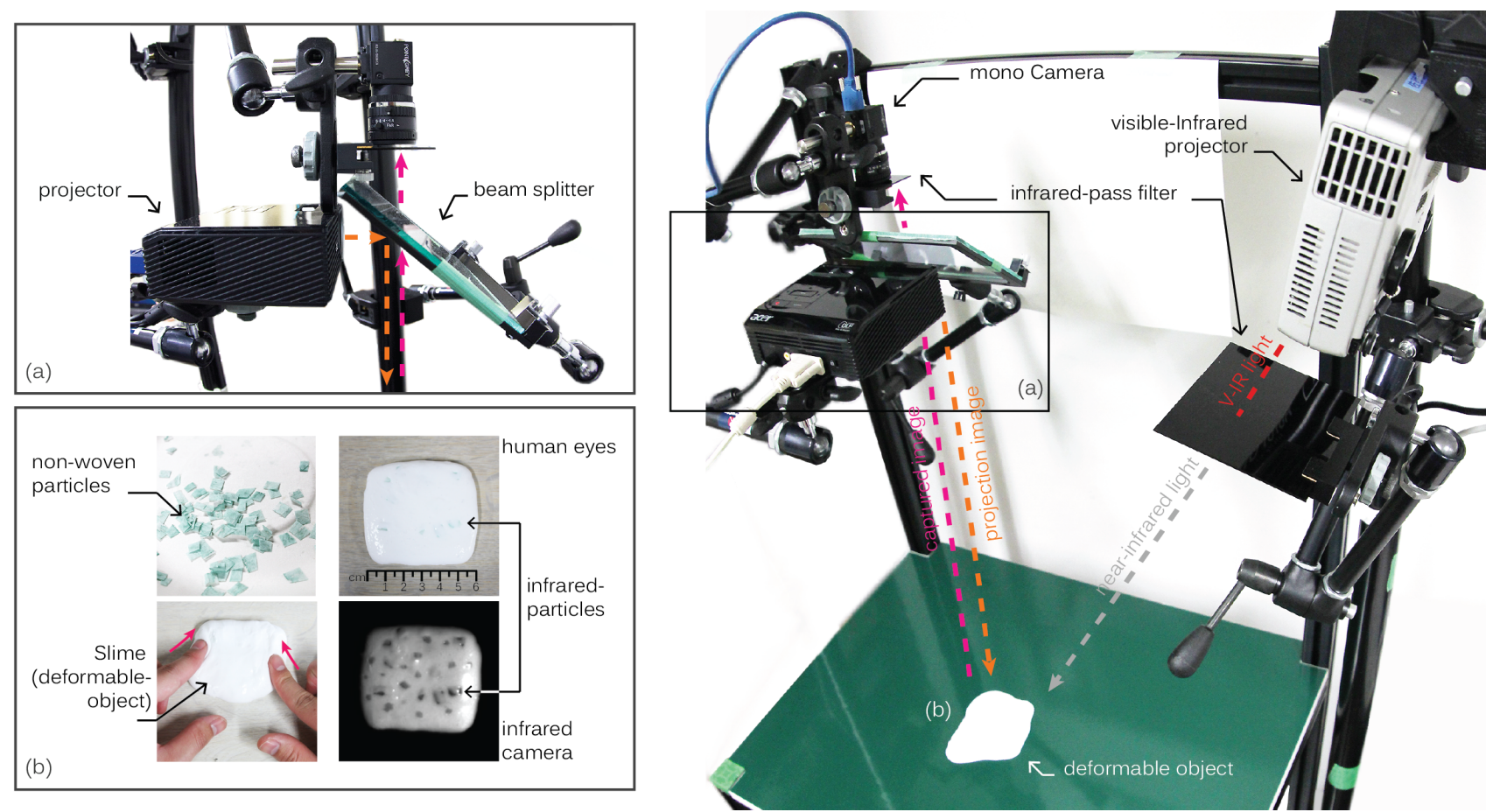

Fig. 7 Experimental setup. The visible-IR projector with IR-pass filter is used as an IR light source. (a) The projector and camera are placed in the same plane using a beam splitter; (b) slime (viscoelastic material) with embedded IR-based particles is used as the deformable object in our experiments.

\section{Experiments}

We conducted both objective and subjective experiments to evaluate the proposed method.

\subsection{System configuration and demonstration}

Figure 7 shows the hardware configuration of our prototype. We place a projector (ACER K10) and a camera (PointGrey Flea3 FL3-U3-13S2M-CS) so that they share the same optical axis using a beam splitter (Edmund Optical VIS Plate, $1.25 \mathrm{~cm}$ ). The camera is equipped with an IR-pass filter (Edmund Optical Cast Plastic IR $700 \mathrm{~nm}$ Long-pass Filter). A visible-IR projector (PLUS V-1100) with an IR-pass filter (same as the camera's filter) is used as a spatially uniform infrared light source.

The images of the deformable object are acquired at a resolution of $640 \times 480$ pixels. The image acquisition process, motion measurement, and deformation estimation run on the same PC (CPU: Intel Core i7 950 3.07 GHz, RAM: 6GB, GPU: NVIDIA GeForce GT520 2GB). The within-two-frames feature tracking method is implemented on the GPU. We divide the surface ROI into $3 \times 3$ blocks (i.e., $m=3$, in Section 3.2). The camera and projector share an optical axis; thus, we use homography for the coordinate transformation between the camera and projector.
We use an approximately $36 \mathrm{~cm}^{2}$ piece of slime (shown in Fig. 7(b)) as a deformable material in all experiments. The material is made by mixing sodium borate with polyvinyl alcohol. It exhibits both viscosity and elasticity, and provides global and local deformations. The material flows like a liquid for a long period, but it has a short period of rigidity. We embed the IR ink-soaked fabric particles into the deformable object's surface, as described in Section 4.

Figure 8(a) shows the self-dilatation deformation of the surface. The projected graphics become larger according to the self-dilatation. Figures 8(b) and 8(c) show the deformation of the projected results when a user interacts with the surface. The projected graphics deform according to the user's tangential deformation manipulation.

\subsection{Accuracy evaluation}

As described in Section 3, the proposed deformation estimation method is designed to avoid tracking errors that result from occluded features. We evaluate how accurately the proposed method estimates the deformation when feature points are sometimes occluded in a sequence by comparing our results with a reference.

The reference is obtained by manual feature tracking through a whole sequence using the procedure de- 

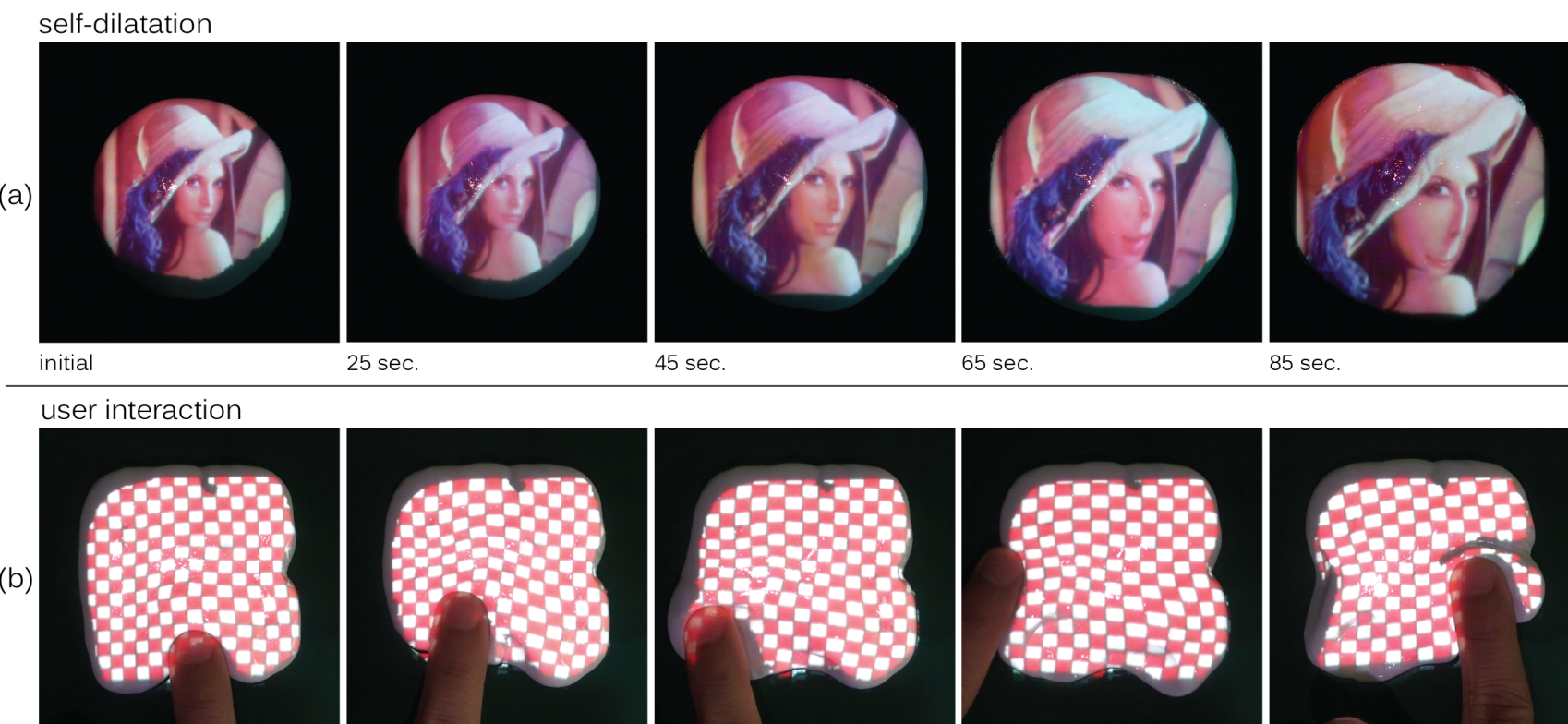

$25 \mathrm{sec}$.

45 sec.

65 sec.

$85 \mathrm{sec}$.
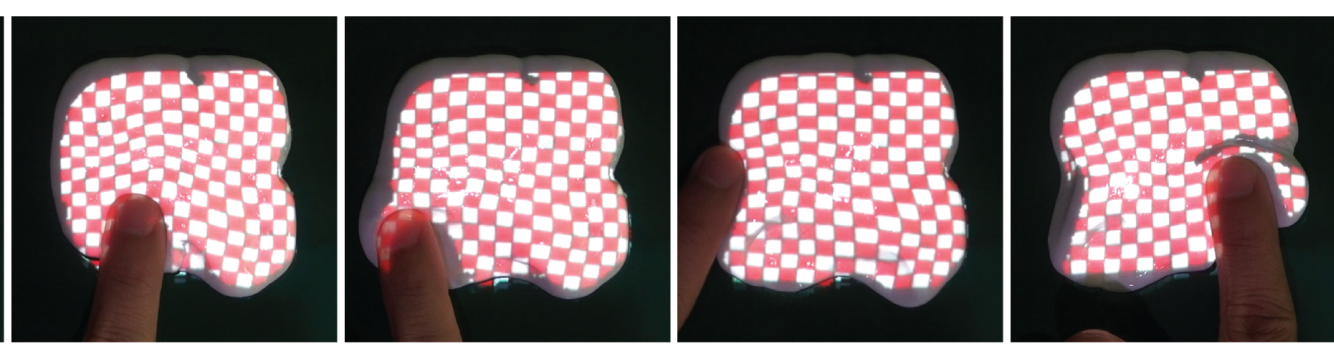

00:17 min.

00:32 min

00:39 min

01:14 min

02:07 $\mathrm{min}$

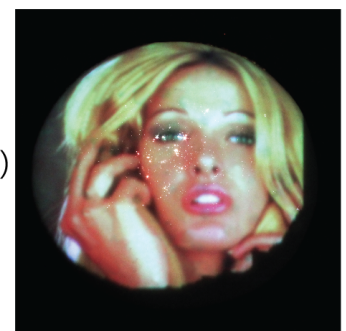

00:00 min.

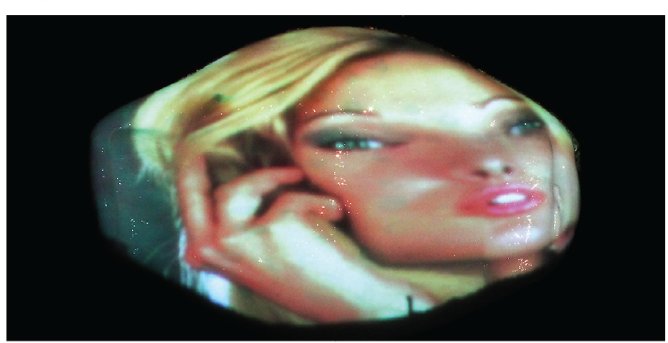

00:19 min.

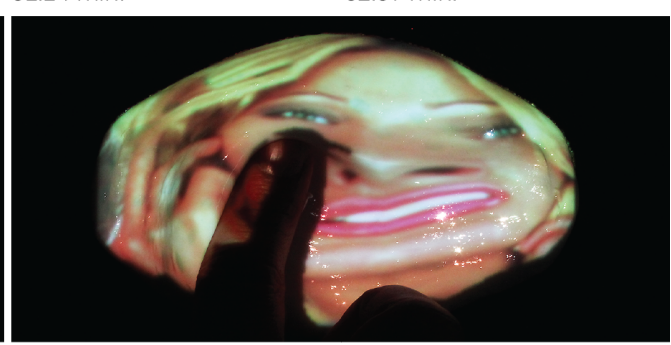

01:03 min.

Fig. 8 Preliminary results of the implementation. The different sets of selected frames show the visualization of the surface self-dilatation deformation (a) and user interaction (b, c). Users can interact with a deformable surface by kneading, touching, pulling (b), and stretching (c). The projected graphics were deformed as closely as possible to the deformed surface.

scribed below. The experimenter selects feature points in the first captured frame and begins tracking them in the next captured frame. If there are occluded features, the experimenter does not attempt to track them in that frame. When such features reappear, the experimenter recovers these previously occluded features and continues to track them. The feature tracking results are then processed using the MLS method to estimate the deformation, which is the same as the proposed method.

In the experiment, we used 1.25 features per $\mathrm{cm}^{2}$ or a total 45 feature points for both the proposed and reference methods. The experimenter caused deformations by pushing the surface. A video sequence was recorded by the system's IR camera. The same sequence was used for both the proposed and reference methods.

The estimated positions of grid vertices used in the MLS method are plotted in Fig. 9. The proposed method (blue) is compared with the reference (orange) at 225 vertices. From the figure, we confirm that the trajectories in our method and the reference method look sim-
Table 1 Results of the user test.

\begin{tabular}{c|c} 
Number of features $\left(\right.$ per $\left.\mathrm{cm}^{2}\right)$ & Average rating \\
\hline 0.42 & 3.1 \\
0.83 & 4.2 \\
1.25 & 4.4
\end{tabular}

ilar. On average, the Euclidean distance between the estimation results of our method and those of the reference is only 5.4 pixels, which corresponds to approximately $0.14 \mathrm{~cm}$ on the surface. Moreover, the correlation coefficient analysis shows that the results of our method and the reference are similar $(\rho=0.98)$. Consequently, we can confirm that our method performed the surface deformation estimation with comparable accuracy to the reference method using manual feature tracking.

\subsection{User test}

Our system is designed to be used by a user; thus, we must assess the system from the user's perspective. 

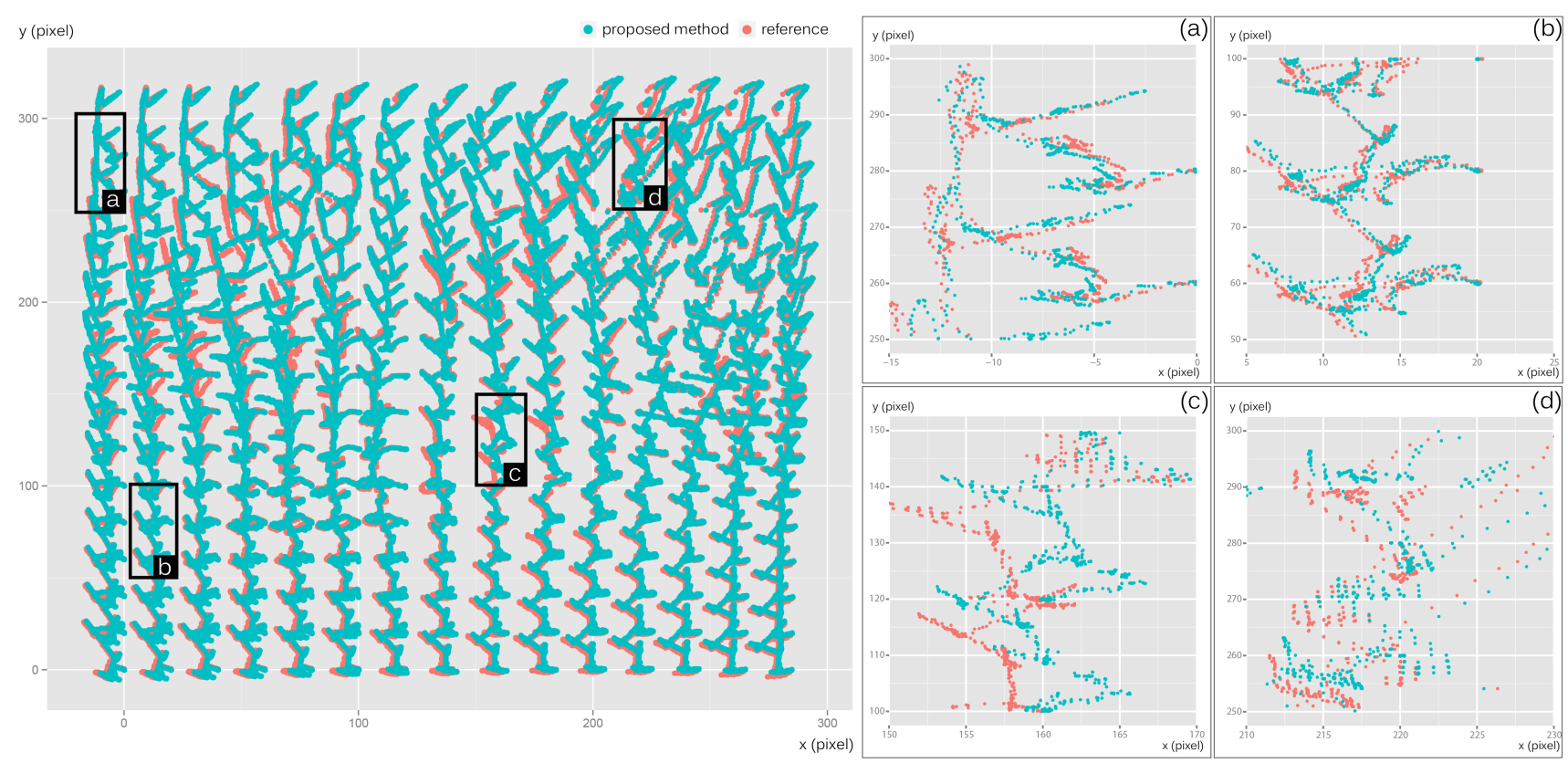

Fig. 9 Estimation results for our method (blue) compared with those of the reference method (orange) for 225 vertices.
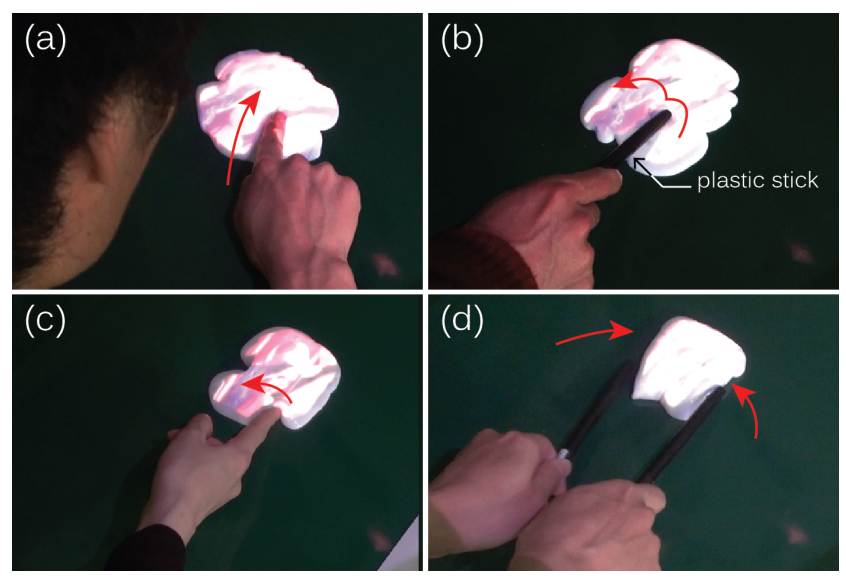

Fig. 10 User experiment interactions: (a) pushing, (b) touching, (c) pulling, and (d) pinching.

Here, we show the results from our user study, in which participants subjectively evaluate how realistic the visualization results of our proposed system are.

In the experiment, each participant is asked to interact with the deformable surface under three conditions, i.e., $0.42,0.83$, and 1.25 features per $\mathrm{cm}^{2}$ or a total 15, 30, and 45 feature points, respectively, are used for the estimation. For each condition, participants perform four types of interactions: pinching, kneading, pushing, and shrinking (using their hand, or using plastic sticks) (Fig. 10). The order of the specified interactions is random for each participant. After the experiment, we ask each participant to rate the deformation visualization result subjectively. In particular, we ask them the following question: "How realistic do you think the graphics projected on the deformable surface were?". The question is asked for each experimental condition. The participants answer the question using a 5-point Likert scale, where 1 is rather unrealistic and 5 is very realistic.

Eleven subjects (9 males, 2 females) between the ages of 22 and 25 participated in the experiment. Table 1 shows the average subjective ratings. For 0.83 or more features per $\mathrm{cm}^{2}$, the participants found the deformation results to be satisfactory (average $>4.2$ ). However, 0.42 features per $\mathrm{cm}^{2}$ were less effective in the estimation of the surface deformation (average $=3.1$ ). The Likert scale rating was analyzed using the Friedman test. There was a statistically significant difference in the realistic deformation estimation depending on the number of feature points per $\mathrm{cm}^{2}, \chi^{2}=16.06$ $(p<0.05)$. Post-hoc pairwise comparisons were conducted using the Wilcoxon signed-rank test. For posthoc analyses, we applied the Bonferroni correction for multiple comparisons to a significance level of 0.017 (i.e., $p=0.05 / 3$ multiple comparisons). There were statistically significant differences in the realistic deformation estimation between 0.42 and 0.83 features per $\mathrm{cm}^{2}$ $(Z=-2.76, p<0.01)$ and between 0.42 and 1.25 features per $\mathrm{cm}^{2}(Z=-2.89, p<0.01)$. However, there was no significant difference between 0.83 and 1.25 features per $\mathrm{cm}^{2}(Z=-0.71, p=0.48)$.

In summary, the experimental results prove that the number of features per $\mathrm{cm}^{2}$ has a significant effect on the realistic deformation estimation result. Increasing the number of feature points improves the realistic es- 


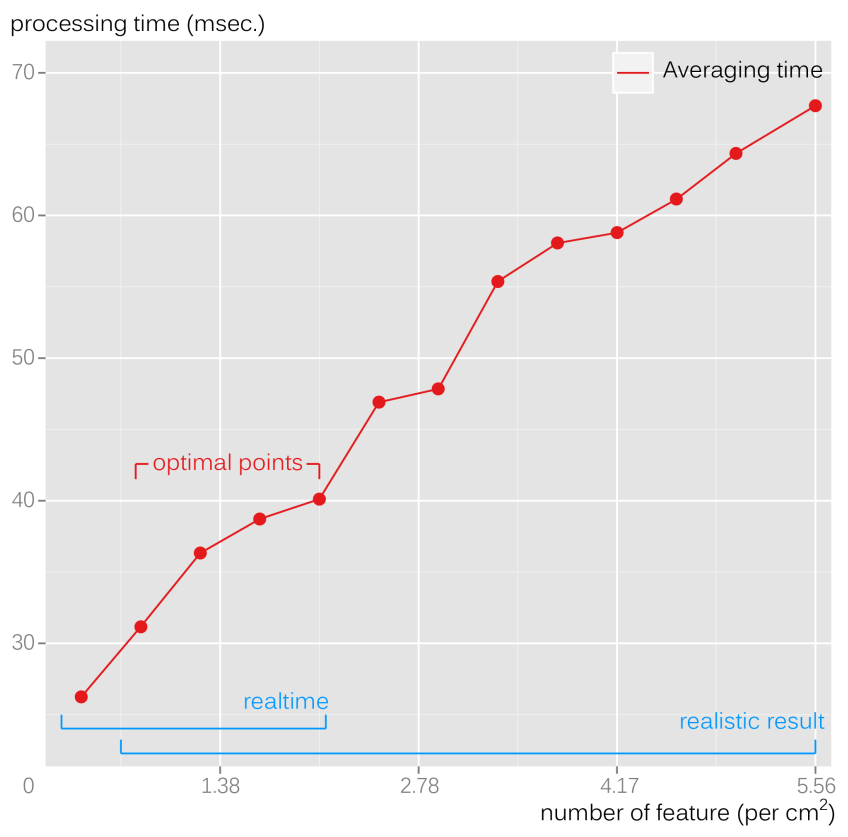

Fig. 11 Average processing times using 0.42 - 5.56 feature points per square centimeter.

timation results. In particular, 0.83 and 1.25 features per $\mathrm{cm}^{2}$ clearly provide a more realistic deformation visualization than 0.42 features per $\mathrm{cm}^{2}$.

\subsection{Optimal number of features}

Although the number of feature points has significant effect on the realistic deformation visualization result, as confirmed in Section 5.3, increasing the number of feature points requires a longer computational time. We aim to apply our method to interactive applications; thus, we must consider the trade-off between real-time performance and the number of feature points necessary for realistic deformation estimation.

We conducted an experiment to find the appropriate balance between realistic deformation visualization and real-time performance. The computation time of the system was evaluated by increasing the number of feature points. We tried to find the optimal number of feature points for realistic deformation estimation within real-time performance.

The average processing times for 300 frame iterations with 13 different feature density per $\mathrm{cm}^{2}(0.42$, $0.83,1.25,1.66,2.03,2.43,2.84,3.24,3.65,4.05,4.46$, 5.00 , and 5.56) are shown in Fig. 11. The processing time exceeded real-time performance requirements (at 25 frames per second) when the feature density was greater than 2.03. The deformation estimation result was sufficiently realistic to satisfy users when the feature density was 0.83 and above (Section 5.3). In other words, the optimal number of feature points that balances realistic deformation visualization within realtime performance was $0.83-2.03$ in this prototype system.

\section{Discussion}

Our technique allows an intuitive MR experience in projection-based technologies using a simple hardware setup that includes a projector, an IR camera, and an IR light source. The proposed method is not limited by the characteristics of deformable materials (e.g., elastic, viscous, or viscoelastic). Such limitations can be overcome by painting the object's surface with IR ink or by embedding pieces of fabric soaked in the ink into the surface. Owing to a state-of-the-art deformation estimation technique (i.e., MLS), our method uses a relatively small number of feature points to approximate deformations for large-scale surfaces. Here, we discuss the potential applications and limitations of the proposed system.

Potential applications: Adding a deformable projected graphics to physical clay materials has many potential educational applications. Clay is often used as an early childhood educational tool. Mixing the advantages of computer-supported education and our deformed projected graphics using a projector-camera system, we can facilitate new interactive educational activities. For example, teachers and students can experience the deformation of physical objects visualized by projection during fluid dynamics experiments. It can also support graphical designers in creating deformable graphics. They can explore their designs easily by deforming clays or other deformable materials on which the deformation information is projected. As another application, we can apply the proposed system to toys for small children; they can, for example enjoy deforming preferred graphic images by changing the form of the clay.

Limitations: We have proposed a within-two-frames feature tracking method with the MLS deformation reconstruction technique to estimate the deformation of a non-textured surface for projection-based MR technologies. With the proposed technique, the projection graphics can deform to match the real object, even for self-dilating objects made of viscoelastic materials. However, there are currently some limitations. First, although our method can estimate surface deformation without any mechanical devices, artificial textures, which are either painted on or embedded into the physical object's surface, are required. However, we do not 
believe that this leads to any critical problems. The textures are invisible to the human eye; thus, they do not degrade the image quality of the augmented graphics. Second, the proposed method shares a limitation regarding deformation estimation with other vision-based methods. As with other methods, estimating twist deformation is not possible because the motion detected by feature tracking does not include rotational information. Although this could be solved by applying a more complex marker, e.g., an identifiable $2 \mathrm{D}$ visual marker, such a marker would disturb the estimation of any deformation smaller than the employed marker. While the dense optical flow technique (Alvarez et al, 2000) might include rotational information, it is limited by the fact that computational resources depend on the size of the surface material for real-time processing. Moreover, the deformation result from interpolated flow can sometimes suffer from tracking noise, leading to lack of realism. In contrast, our sparse tracking method allows size-independent real-time processing. Our method also minimizes the lack of realism by reducing the number of feature points and using interpolated deformation flows, instead.

In this paper, we proposed combining a sparse tracking method with MLS interpolation to realize robust and real-time tangential deformation estimation. We designed our method so that any sparse tracking methods can be used in combination with the MLS. Investigating which sparse tracking method provides the best performance is beyond the scope of this paper, and will be tackled in our future work.

\section{Conclusions}

To the best of our knowledge, no empirical work has addressed projection-based augmentation of nonrigid surfaces based on tangential deformation estimation. This study has described a new technique that can estimate the deformation of a non-textured surface successfully. The system combines the within-two-frames motion measurement and the MLS method for tangential deformation estimation. The results show the effectiveness of our deformation estimation method compared with manual feature tracking. Determining the optimal number of feature points indicated that it is possible to balance realistic deformation visualization and realtime performance. In addition, we presented a range of applications which can benefit from the projectionbased technology developed by us. In the future, we will extend the proposed method, which currently only supports 2D deformation, to augment 3D surface deformation by applying multiple cameras and projectors.

\section{References}

Alvarez L, Weickert J, Snchez J (2000) Reliable estimation of dense optical flow fields with large displacements. International Journal of Computer Vision 39(1):41-56

Bandyopadhyay D, Raskar R, Fuchs H (2001) Dynamic shader lamps: painting on movable objects. In: Proceedings of the IEEE/ACM International Symposium on Augmented Reality, pp 207-216

Bimber O, Iwai D (2008) Superimposing dynamic range. ACM Transactions on Graphics 27(5):15:115:8

Bimber O, Raskar R (2005) Spatial Augmented Reality: Merging Real and Virtual Worlds. A. K. Peters, Ltd.

Bluteau J, Kitahara I, Kameda Y, Noma H, Kogure K, Ohta Y (2005) Visual support for medical communication by using projector-based augmented reality and thermal markers. In: Proceedings of the International Conference on Artificial Reality and Telexistence, pp 98-105

Chang RC, Tseng FC (2010) Automatic detection and correction for glossy reflections in digital photograph. In: 3rd IEEE International Conference on Ubi-media Computing (U-Media), 2010, pp 44-49

Follmer S, Johnson M, Adelson E, Ishii H (2011) deform: An interactive malleable surface for capturing $2.5 \mathrm{~d}$ arbitrary objects, tools and touch. In: Proceedings of the 24th Annual ACM Symposium on User Interface Software and Technology, ACM, New York, NY, USA, UIST '11, pp 527-536

Fujimoto Y, Smith R, Taketomi T, Yamamoto G, Miyazaki J, Kato H, Thomas B (2014) Geometrically-correct projection-based texture mapping onto a deformable object. Visualization and Computer Graphics, IEEE Transactions on 20(4):540-549

Haouchine N, Dequidt J, Kerrien E, Berger MO, Cotin S (2012) Physics-based augmented reality for 3d deformable object. In: Workshop on Virtual Reality Interaction and Physical Simulation

Heo S, Lee G (2013) Indirect shear force estimation for multi-point shear force operations. In: Proceedings of the SIGCHI Conference on Human Factors in Computing Systems, ACM, New York, NY, USA, CHI '13, pp 281-284

Hisada M, Yamamoto K, Kanaya I, Sato K (2006) Freeform shape design system using stereoscopic projector - hyperreal 2.0. In: SICE-ICASE International Joint Conference, pp 4832-4835

Ito Y, Kim Y, Obinata G (2014) Acquisition of contact force and slippage using a vision-based tactile sensor with a fluid-type touchpad for the dexterous 
handling of robots. Advances in Robotics \& Automation 3(116)

Iwai D, Sato K (2010) Document search support by making physical documents transparent in projection-based mixed reality. Virtual Reality 15(23):147-160

Johnson MK, Cole F, Raj A, Adelson EH (2011) Microgeometry capture using an elastomeric sensor. ACM Trans Graph 30(4):46:1-46:8

Jones BR, Benko H, Ofek E, Wilson AD (2013) Illumiroom: peripheral projected illusions for interactive experiences. In: Proceedings of the ACM Annual Conference on Human Factors in Computing Systems, pp 869-878

Kamiyama K, Vlack K, Mizota T, Kajimoto H, Kawakami N, Tachi S (2005) Vision-based sensor for real-time measuring of surface traction fields. IEEE Computer Graphics and Applications 25(1):68-75

Kocev B, Ritter F, Linsen L (2013) Projector-based surgeon-computer interaction on deformable surfaces. International Journal of Computer Assisted Radiology and Surgery 8(6):1015-1025

Matoba Y, Sato T, Takahashi N, Koike H (2012) Claytricsurface: an interactive surface with dynamic softness control capability. In: ACM SIGGRAPH Emerging Technologies, p 6:1

Mine M, van Baar J, Grundhofer A, Rose D, Yang B (2012) Projection-based augmented reality in disney theme parks. IEEE Computer 45(7):32-40

Ni T, Karlson AK, Wigdor D (2011) AnatOnMe: facilitating doctor-patient communication using a projection-based handheld device. In: Proceedings of ACM SIGCHI Conference on Human Factors in Computing Systems, pp 3333-3342

Pilet J, Lepetit V, Fua P (2008) Fast non-rigid surface detection, registration and realistic augmentation. International Journal of Computer Vision 76(2):109122

Piper B, Ratti C, Ishii H (2002) Illuminating clay: A 3-d tangible interface for landscape analysis. In: Proceedings of ACM SIGCHI Conference on Human factors in Computing Systems, pp 355-362

Raskar R, Welch G, Low KL, Bandyopadhyay D (2001) Shader lamps: Animating real objects with imagebased illumination. In: Proceedings of the Eurographics Workshop on Rendering, pp 89-102

Raskar R, Ziegler R, Willwacher T (2006) Cartoon dioramas in motion. In: Proceedings of the International Symposium on Non-Photorealistic Animation and Rendering, pp 7-12

Rivers A, Adams A, Durand F (2012) Sculpting by numbers. ACM Transactions on Graphics 31(6):157:1-157:7
Saakes D, Chiu K, Hutchison T, Buczyk BM, Koizumi N, Inami M, Raskar R (2010) Slow display. In: ACM SIGGRAPH Emerging Technologies, p 22

Sato T, Mamiya H, Koike H, Fukuchi K (2009) PhotoelasticTouch: transparent rubbery tangible interface using an LCD and photoelasticity. In: Proceedings of the 22nd annual ACM symposium on User interface software and technology - UIST '09, ACM Press, New York, New York, USA, pp 43-50

Schaefer S, McPhail T, Warren J (2006) Image deformation using moving least squares. ACM Transactions on Graphics 25(3):533-540

Shi J, Tomasi C (1994) Good features to track. In: Proceedings of IEEE Conference on Computer Vision and Pattern Recognition, pp 593-600

Shimazu S, Iwai D, Sato K (2011) 3d high dynamic range display system. In: Proceedings of the 10th IEEE/ACM International Symposium on Mixed and Augmented Reality, pp 235-236

Shimizu N, Yoshida T, Hayashi T, de Sorbier F, Saito H (2013) Non-rigid Surface Tracking for Virtual Fitting System. In: International Conference on Computer VIsion Theory and Applications, 2013, pp 1-7

Steimle J, Jordt A, Maes P (2013) Flexpad: highly flexible bending interactions for projected handheld displays. In: Proceedings of ACM SIGCHI Conference on Human Factors in Computing Systems, pp 237246

Uchiyama H, Marchand E (2011) Deformable random dot markers. In: Proceedings of IEEE International Symposium on Mixed and Augmented Reality, pp $237-238$

Uchiyama H, Saito H (2011) Random dot markers. 2014 IEEE Virtual Reality (VR) 0:35-38

Vlack K, Mizota T, Kawakami N, Kamiyama K, Kajimoto H, Tachi S (2005) GelForce: a vision-based traction field computer interface. In: CHI '05 Extended Abstracts on Human Factors in Computing Systems, ACM, New York, NY, USA, pp 1154-1155

Wu HY, Rubinstein M, Shih E, Guttag J, Durand F, Freeman W (2012) Eulerian video magnification for revealing subtle changes in the world. ACM Transactions on Graphics 31(4):65-72 